\title{
Developing New Routine for Processing Two-Dimensional Coincidence Doppler Energy Spectra and Evaluation of Electron Subsystem Properties in Metals
}

\author{
P.S. Stepanov ${ }^{a, *}$, S.V. Stepanov ${ }^{b, c, d}$, V.M. Byakov ${ }^{b, d, e}$, F.A. Selim ${ }^{a}$ \\ ${ }^{a}$ Center for Photochemical Sciences, Bowling Green State University, OH, USA \\ ${ }^{b}$ NRC "Kurchatov Institute" - Institute for Theoretical and Experimental Physics, \\ B. Cheremuskinskaya st., 25. 117218 Moscow Russia \\ ${ }^{c}$ National Research Nuclear University "MEPhI", 115409, Moscow, Russia \\ ${ }^{d}$ Lomonosov Moscow State University, Chemical Department, Leninskie Gory, Moscow, Russia \\ ${ }^{e}$ D. Mendeleyev University of Chemical Technology of Russia, Miusskaya sq., 9, 125047, Moscow, Russia
}

A digital coincidence Doppler broadening (CDB) spectrometer consisting of two HPGe detectors is assembled; the energy resolution of each detector is $1.1 \mathrm{keV}$ at energies near the annihilation line. Two desktop applications for CDB spectra processing are developed. TLIST Processor converts two-dimensional CDB spectra into onedimensional spectra. Another tool SW Calculator deconvolutes the experimental CDB spectra into contributions from positron annihilation with valence, core and conduction band electrons. The program estimates the energies of the corresponding electrons and evaluates $S$ and $W$ parameters of the CDB spectra.

DOI: 10.12693/APhysPolA.132.1628

PACS/topics: Positron annihilation, Doppler broadening, metals, core electrons

\section{Introduction}

During the last five decades positron annihilation spectroscopy (PAS) has been widely applied in material science (metals, semiconductors, polymers) [1], radiation chemistry [2], biology and medicine [3]. Coincidence Doppler broadening (CDB) spectroscopy is a powerful PAS technique, highly sensitive to the presence of defects and sensitive to the momentum distributions of core and valence electrons in a wide range of materials [4].

In CDB experiments the energies $E_{1}$ and $E_{2}$ of a pair of annihilation photons related to the annihilation of the same positron are simultaneously registered. CDB spectrum is constructed of the energy pairs $\left(E_{1}, E_{2}\right)$ that satisfy the following condition: $1022 \mathrm{keV}-E_{b}-\delta<$ $E_{1}+E_{2}<1022 \mathrm{keV}+\delta$, where $E_{b}$ is the binding energy of the lowest core shell electrons and $\delta$ is the sum of energy resolutions of both $\gamma$-detectors. The timing resolution of the coincidence spectrometer is about $100 \mathrm{~ns}$, which results in a high signal-to-noise ratio (more than $10^{5}$ ). Unlike the single-detector Doppler broadening spectroscopy, CDB-measurements provide a unique tool to analyze the local chemical environment at the positron annihilation site.

\section{Experimental setup}

A digital coincidence Doppler broadening spectrometer has been installed at Bowling Green State University

\footnotetext{
*corresponding author; e-mail: petrs@bgsu.edu
}

in 2016. It is assembled from two high-purity Ge detectors operating at $4.5 \mathrm{kV}$ and a pair of Lynx multichannel analyzers manufactured by Canberra Industries. Each analyzer supports up to 32768 channels. This results in a channel width of a $60 \mathrm{eV}$. The energy resolution of the detectors is $1.1 \mathrm{keV}$ at $0.5 \mathrm{MeV}$. This value was determined by interpolation of the FWHM change from $1.7 \mathrm{keV}$ at $1173 \mathrm{keV}\left({ }^{60} \mathrm{Co}\right)$ to $1.26 \mathrm{keV}$ at $660 \mathrm{keV}\left({ }^{137} \mathrm{Cs}\right)$. The preamplifier signal width is $1 \mu \mathrm{s}$. Maximum preamplifier count rate performance is $2 \times 10^{5}$ counts $/ \mathrm{s}$. When the detector collects energy, a preamplifier converts the signal charge into a readable step-function pulse, where the amplitude is proportional to the total charge accumulated during an event.

The novelty of Canberra's spectrometer is absence of the coincidence unit. Analyzers are connected to each other and share a single internal clock. Every device continuously saves independent series of energy and time events. The two-dimensional energy spectrum is reconstructed on-the-fly during acquisition with Canberra's TLIST Scanner software. This application imports timestamped energy data files from both analyzers, matches energy values registered within a time interval of $100 \mathrm{~ns}$ and reconstructs two-dimensional energy histogram.

Activity of the ${ }^{22} \mathrm{Na}$ positron source used in this $\mathrm{CDB}$ spectrometer is about $10 \mu \mathrm{Ci}(0.37 \mathrm{MBq})$. The source $\left({ }^{22} \mathrm{NaCl}\right.$ salt) resides in between of two $8 \mu \mathrm{m}$ Kapton foils sealed with a silicon glue. The source is sandwiched with a pair of identical samples and placed in the middle of two high purity Ge detectors facing towards each other (Fig. 1). The distance between detectors is $30 \mathrm{~cm}$. The preamplifier rate during the measurements 
is $1.2 \times 10^{3} \mathrm{MeV} / \mathrm{s}$ (upper limit is $4 \times 10^{5} \mathrm{MeV} / \mathrm{s}$ ). Detector dead time is $4.5 \%$. The count rate registered by each analyzer is $\sim 3 \times 10^{3}$ counts $/ \mathrm{s}$. Acquisition at such relatively low count rate prevents notable pileup effects and preamplifier saturation. Coincidence count rate is $\sim 50$ counts $/$ s.

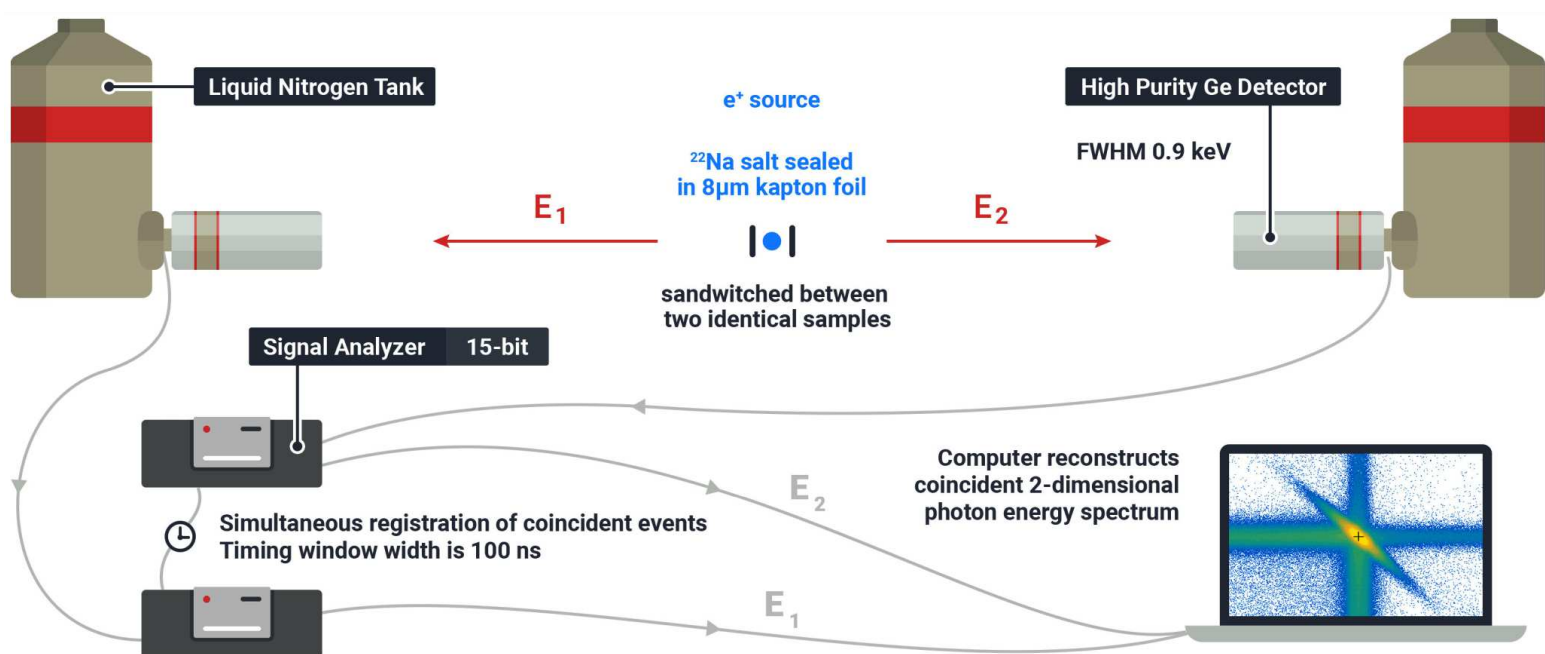

Fig. 1. Scheme of the digital Doppler coincidence spectrometer.

\section{Subtraction of the two-dimensional background from the CDB spectra}

Typically, the background subtraction procedures of the Doppler spectra are performed for one-dimensional CDB histograms [5, 6]. Two-dimensional fits of the full Doppler spectrum were reported by E.D. Nascimento et. al. [7]. In this work we report a development of a desktop software (TLIST Processor) that subtracts twodimensional background from the raw experimental spectra. The software accounts for a number of effects that contribute to the spectrum background.

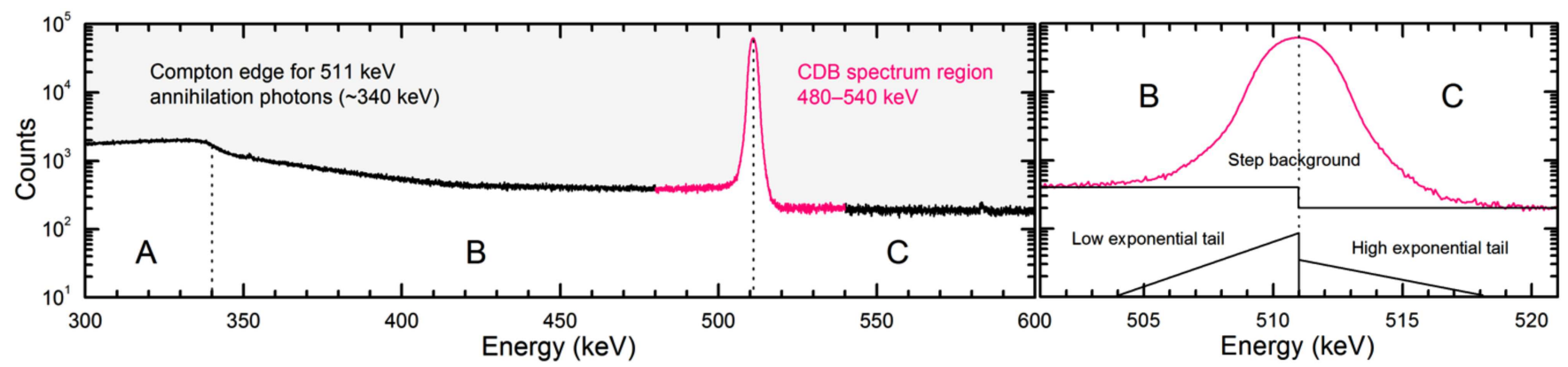

Fig. 2. Single-detector Doppler spectrum of Al. Within the studied fitting region (480-540 keV) the background can be approximated by a sum of a step function and two exponential tails. Sum of the above mentioned contributions is convoluted with the detector resolution functions. In our case it is a Gaussian with the FWHM $\approx 1.1 \mathrm{keV}$.

The Compton scattered 1.27 MeV photons which accompany $\mathrm{e}^{+}$birth in the ${ }^{22} \mathrm{NaCl}$ source give a constant contribution in the $\mathrm{A}, \mathrm{B}$, and $\mathrm{C}$ energy regions (Fig. 2). In between of the Compton edge and the $511 \mathrm{keV}$ photopeak (Fig. 2, region B) we have a number of different effects. Major contribution into this region comes from photons that Compton scattered outside of the active detector volume (in the specimen or in the detector case) [8]. Within the studied fitting energy interval (480$540 \mathrm{keV}$ ) the above mentioned contributions are well approximated by a two-step function [5].

Three-gamma annihilation of positrons contributes into regions $\mathrm{A}$ and $\mathrm{B}$. In fact, small fraction of $\mathrm{e}^{+}$may form positronium atoms (for example, in a silicon glue of the $\mathrm{e}^{+}$source). The application we developed can approximate this three-gamma contribution profile with the Ore-Powell formula [9]. In this work we did not account on this effect because it is considered to be negligible.

Incomplete charge collection in the detectors give rise to the exponential tail in the region $\mathrm{B}$. The pile-up effect (overlapping of pulses from two different photons) in the region $\mathrm{C}$ can be represented by the exponent with a negative slope $[5,10]$.

On the two-dimensional energy spectrum the two ridge profiles are fitted with a sum of the two-step background and two exponential tails convoluted with a detector resolution function. In a perpendicular plane, the ridges are parameterized by a sum of two Gaussian functions with 
different dispersions and intensities.

After the background subtraction the two-dimensional energy spectrum $C\left(E_{1}, E_{2}\right)$ is transformed into onedimensional CDB spectrum (i.e. counts vs. $\Delta=E_{1}-$ $\left.E_{2}\right)$. This procedure provides clean CDB spectrum with almost zero background and allows an adequate extraction of the electron momentum contributions and defect parameters.

The subtracted background contribution in onedimensional CDB spectrum of aluminum is shown in Fig. 3. The $\mathrm{CDB}$ spectrum of $\mathrm{Al}$ (Fig. 3, right) includes contribution of $\mathrm{e}^{+}$annihilation in the Kapton film that seals ${ }^{22} \mathrm{Na}$ source which is about $12 \%$ from total annihilation events.

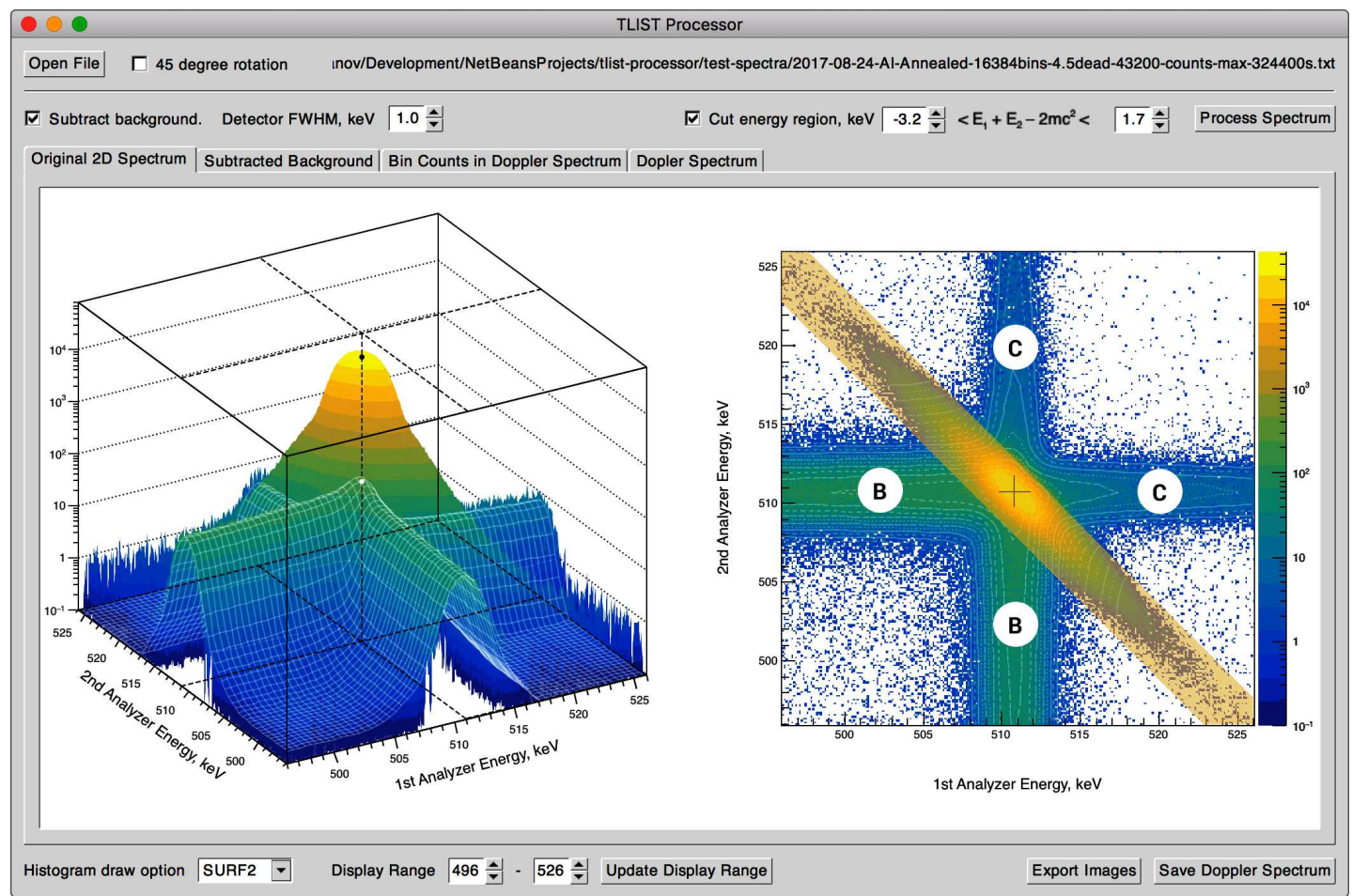

Fig. 3. TLIST Processor application fits and subtracts background from the two-dimensional CDB energy spectrum. Ridge profile for $E>511 \mathrm{keV}$ (a) consists of contribution from Compton scattered $1.27 \mathrm{MeV}$ photons and pile-up effect. In the range $E<511 \mathrm{keV}$ (b) there is a Compton scattered $511 \mathrm{keV}$ photon outside of the active detector volume and incomplete charge collection. Left: convoluted and fitted background is represented by the overlay mash. Right: events from the yellow band of a width $1022 \mathrm{keV}-E_{b}-\delta<E_{1}+E_{2}<1022 \mathrm{keV}+\delta$ are selected to construct single-dimensional CDB spectrum. Here $E_{b}$ stands for the typical binding energy of core electrons, $\delta=1.1 \mathrm{keV}$ is the energy resolution of $\gamma$-detectors.

The developed TLIST Processor is a desktop GUI application written in $\mathrm{C}++$ built with CERN ROOT scientific framework (Fig. 3). The source code is available on GitHub and distributed under GNU Public License 3.0.

To take into account the energy resolution of a CDB spectrometer, the background function is convoluted with two Gaussian functions with FWHMs equal to the energy resolutions of the corresponding detectors.

\section{Reconstruction of the CDB spectra}

Calculation of the shape of CDB spectrum is a complicated quantum-mechanical problem. In order to solve it, we need to know the momentum distribution $n(\boldsymbol{k})$ of the $\mathrm{e}^{+} \mathrm{e}^{-}$pair at a time of annihilation. In most cases, a positron is fully thermalized, so $n(\boldsymbol{k})$ is defined by the momentum distribution of the electrons in the media. Using energy and momentum conservation laws, one may relate the energy difference of annihilation photons with $z$-projection of the momentum (the longitudinal momentum) of the electron that participated in the annihilation process $E_{2}-E_{1} \approx c \hbar k_{z}$. Thereby the shape of a CDB spectrum can be calculated as follows:

$$
C\left(\Delta=E_{1}-E_{2}\right) \propto \int n(\boldsymbol{k}) \delta\left(\Delta-c \hbar k_{z}\right) \mathrm{d}^{3} \boldsymbol{k} .
$$

If $n(\boldsymbol{k})$ possesses spherical symmetry, we may integrate here over the angular variables. It gives:

$$
C(\Delta) \propto \int_{|\Delta| / c \hbar}^{\infty} k n(k) \mathrm{d} k .
$$

Momentum distribution $n(\boldsymbol{k})$ may be approximately reconstructed from the Fourier transforms of the electron wave functions. It is possible to obtain the analytical expression for $n(k)$ only in some simplest cases. It is generally accepted that the conduction band electron wave functions can be represented by plane waves. This leads to the Fermi distribution $n(k) \propto \theta\left(k<k_{\mathrm{F}}\right)$. Its substitution into Eq. (1) gives a contribution to the CDB spectrum of a shape of the inverted parabola $C(\Delta) \propto 1-\left(\Delta / c \hbar k_{\mathrm{F}}\right)^{2}$. Usage of the hydrogen-like ex- 
ponential wave-function for core electrons $\propto \exp (-r / a)$ results in a Lorentzian-like contribution to the spectrum $C(\Delta) \propto\left[1+(a \Delta / c \hbar)^{2}\right]^{-3}$. Obviously, parameter $a$ which determines the decay of the wave function is directly related to the energy of the corresponding electrons: $E_{b}=\operatorname{Ry}\left(a_{\mathrm{B}} / a\right)^{2}$, where $\mathrm{Ry}=13.6 \mathrm{eV}$ and $a_{\mathrm{B}}=0.53 \AA$.

We also applied various expressions for the $\mathrm{e}^{-}$wave functions, namely $\psi_{\mathrm{e}} \propto r \exp (-r / a), \psi_{\mathrm{e}} \propto(r-b)$ $\exp (-r / a)$, and some other functions. For each of them it is possible to calculate analytically corresponding contributions to the CDB spectrum.

\section{Deconvolution of the experimental CDB spectra}

In order to check the goodness of the data acquired by the CDB spectrometer and the developed software, we measured CDB spectra of the annealed metals $\mathrm{Al}, \mathrm{Cu}$ and Fe. For fitting the spectra, we developed a special software (SW Calculator), which deconvolutes one-dimensional coincidence Doppler spectra into separate contributions from valence, core and conduction band electrons according to the simple theoretical models mentioned above. Source code can be downloaded for free from GitHub. The software accounts for a certain energy resolution of the HPGe detectors:

$$
\begin{aligned}
& \text { Counts }\left(\varepsilon_{1}-\varepsilon_{2}\right) \propto \\
& \qquad \iint C\left(E_{1}-E_{2}\right) G\left(\varepsilon_{1}-E_{1}\right) G\left(\varepsilon_{2}-E_{2}\right) \mathrm{d} E_{1} \mathrm{~d} E_{2},
\end{aligned}
$$

where $G$ is the Gaussian energy resolution function with the FWHM of $1.1 \mathrm{keV}$.

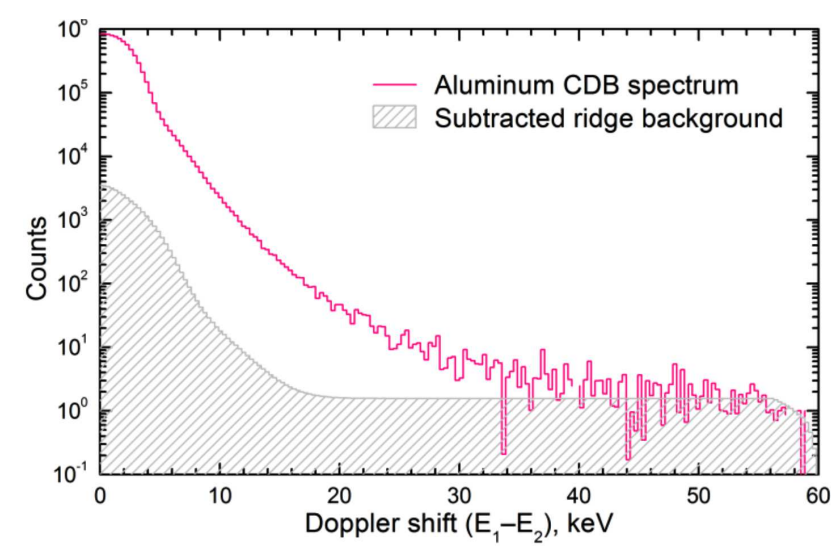

Fig. 4. Contribution of the background events to the experimental CDB spectrum.

A CDB spectrum of $\mathrm{Al}\left(1 s^{2} 2 s^{2} 2 p^{6} 3 s^{2} 3 p^{1}\right)$ deconvoluted into a set of contributions is shown in Fig. 4. Elementary electron Fermi gas theory predicts the following relation between the Fermi energy and the concentration of the conduction electrons [11]:

$$
E_{\mathrm{F}}=\operatorname{Ry}\left(3 \pi^{2} n_{e} a_{\mathrm{B}}^{3}\right)^{2 / 3} .
$$

If we adopt that the conduction electron number density is $n_{e}=3 / \Omega_{\mathrm{Al}}$, (3 is the number of outer electrons $3 s^{2}$ $3 p^{1}$, which are transferred to the conduction band, and
$\Omega_{\mathrm{Al}}=16.7 \AA^{3}$ is the volume per one atom in $\mathrm{Al}$ ), with a help of Eq. (4) we reproduce well the reference value for the $\mathrm{Al}$ Fermi energy $E_{\mathrm{F}}=11.7 \mathrm{eV}$ [11]. The contribution of these conduction electrons to $C(\Delta)$ is described by the inverted parabola. Inner shell electrons $\left(1 s^{2} 2 s^{2} 2 p^{6}\right)$ have following binding energies $1564 \mathrm{eV}, 121 \mathrm{eV}$ and 77 $\mathrm{eV}$. In this work we approximate the inner electron wave functions with Gaussian functions which gives a crude estimation of the electron binding energies. Their contributions may be approximated by three Gaussians as shown in Fig. 5.

\section{Results and discussion}

Experimental Doppler spectra of aluminum, copper and zinc were acquired and processed by the software; they are displayed in Fig. 5. The spectrum of annihilation in Kapton was measured and subtracted from every metal spectrum with a certain intensity obtained from positron annihilation lifetime measurements $(\sim 12 \%)$. Corresponding electron energy values obtained from the fit are displayed in Table I.

TABLE I

Obtained values of the Fermi energies and electron binding energies of three inner electrons (wave functions approximated by Gaussians). Values $\left({ }^{a}\right)$ for the Fermi energy $E_{\mathrm{F}}$ are taken from Ref. [11]. Theoretical values $\left({ }^{b}\right)$ for electrons binding energy $E_{b} n$ are taken from Ref. [12]. Integration ranges for $S$ and $W$ parameters $[\mathrm{keV}]$ are following:

\begin{tabular}{|c|c|c|c|c|}
\hline & $\mathrm{Al}$ & $\mathrm{Fe}$ & $\mathrm{Cu}$ & $\mathrm{Zn}$ \\
\hline \multirow{2}{*}{$E_{\mathrm{F}},[\mathrm{eV}]$} & $12.3(5)$ & $9.36(8)$ & $7.6(4)$ & $9.36(6)$ \\
\hline & $11.7^{a}$ & $11.1^{a}$ & $7.00^{a}$ & $9.47^{a}$ \\
\hline \multirow{2}{*}{$E_{b} 1,[\mathrm{eV}]$} & $40(8)$ & $24.89(8)$ & $23(6)$ & $31.2(5)$ \\
\hline & - & $59^{b}$ & $80^{b}$ & $91^{b}$ \\
\hline \multirow{2}{*}{$E_{b} 2,[\mathrm{eV}]$} & $80(30)$ & $65.6(1.1)$ & $46(13)$ & $75.9(2.1)$ \\
\hline & $77^{b}$ & $61^{b}$ & $82^{b}$ & $94^{b}$ \\
\hline \multirow{2}{*}{$E_{b} 3,[\mathrm{eV}]$} & $215(200)$ & $250(9)$ & $250(60)$ & $309(6)$ \\
\hline & $121^{b}$ & $98^{b}$ & $127^{b}$ & $141^{b}$ \\
\hline$S$ & $0.5531(2)$ & $0.4341(3)$ & $0.4839(4)$ & $0.5121(2)$ \\
\hline$W$ & $0.0201(2)$ & $0.0654(1)$ & $0.0565(1)$ & $0.0524(1)$ \\
\hline
\end{tabular}
$S[-1.6,1.6], W 1[-11.52,-5.52], W 2[5.52,11.52]$. Statistical uncertainties are given in parenthesis.

The uncertainties (given in parenthesis) in the table arise from resolution function uncertainty. During the fit the resolution function FWHM was a free parameter. The energy resolution of the detectors and its relation to the geometry of the experiment, count rate and analyzer binning should be studied more thoroughly.

Fermi energies obtained from the fit are in a good agreement with the reported values [11]. Reasonable agreement by the order of magnitude is found for the electron binding energies [12].

We are looking forward to apply more realistic expressions for the inner electron wave functions including angular dependencies. These improvements are expected to give better agreement for valence and core electron binding energies. 

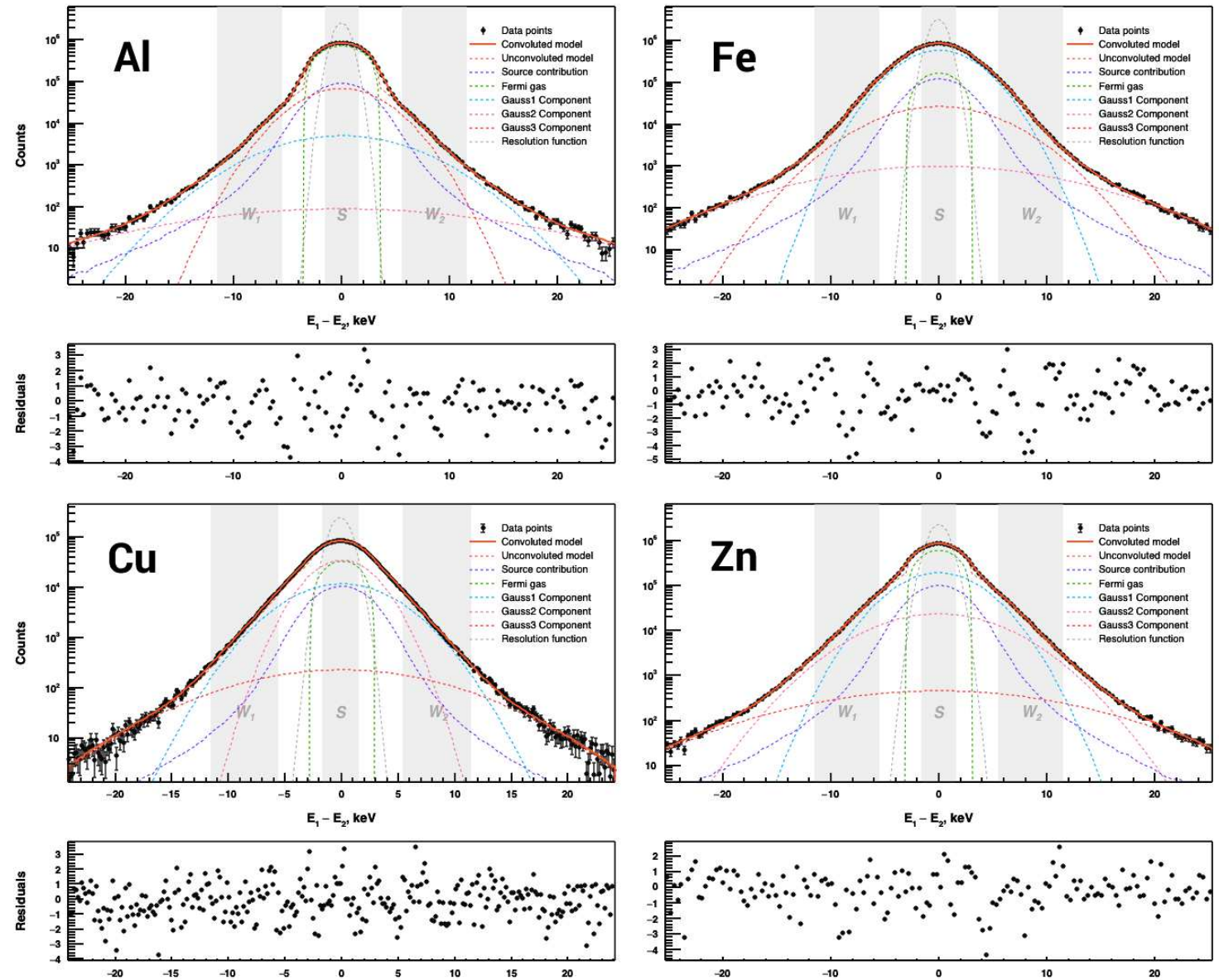

Fig. 5. Doppler spectra of simple metals deconvoluted into a set of contributions of annihilation on Fermi gas electrons (inverted parabola) and Gaussian components. Contribution from annihilation in the positron source is subtracted from spectra. Convolution with the energy resolution function of $1.1 \mathrm{keV} \mathrm{FWHM}$ is applied.

Later on we plan to develop in the software simple description of a positron in structural defects (vacancies, dislocations). Trapped positron can be considered as a particle in a potential well and its contribution into the CDB spectra can be reconstructed. We also plan to proceed with a simultaneous fitting of PALS and CDB spectra. This will result in a better understanding of the defect structure of the studied material.

\section{Conclusions}

Fully-digital Doppler spectrometer with a software coincidence unit, 15-bit analyzers and energy resolution of about $1 \mathrm{keV}$ is set up and tested at Bowling Green State University. A software that fits, subtracts the twodimensional background from the Doppler energy spectrum and constructs one-dimensional CDB spectrum is developed and available for public use. A simple approach for deconvolution of the one-dimensional coincidence Doppler spectra into corresponding contributions from annihilation on conduction band and inner shell electrons is proposed. This theoretical model is implemented in another desktop software that processes onedimensional CDB spectra, calculates $\mathrm{S}$ and $\mathrm{W}$ parameters, extracts Fermi energies of the conduction electrons and binding energies of inner electrons. Experimental spectra of $\mathrm{Al}, \mathrm{Fe}, \mathrm{Cu}$ and $\mathrm{Zn}$ were acquired and processed. Fermi energies are in a good agreement with the reference values.

\section{References}

[1] R. Krause-Rehberg, H.S. Leipner, Springer Series in Solid-State Sciences Positron Annihilation in Semiconductors, vol. 49, (1999).

[2] O.E. Mogensen, Springer Series in Chemical Physics Positron Annihilation in Chemistry vol. 15, (1995).

[3] M. Conti, Physica Medica 25, 1 (2009).

[4] F. Tuomisto, I. Makkonen, Reviews of Modern Physics 85, 1583 (2013).

[5] M. Haaks, T. Staab, K. Maier, Nucl. Instr. Meth. Phys. Res. A 569, 829 (2006).

[6] C.Y. Xi, B.J. Ye, W. Kong, H.M. Weng, X.Y. Zhou, R.D. Han, Meas. Sci. Techn. 16, 1212 (2005).

[7] E.D. Nascimento, V.R. Vanin, N.L. Maidana, O. Helene, J. Phys.: Conf. Ser. 443, 012024 (2013).

[8] P. Pikart, C. Hugenschmidt, Nucl. Instr. Meth. Phys. Res. A 750, 61 (2014). 
[9] V.B. Beresteckij, E.M. Lifšic, L.P. Pitaevskij, Quantum electrodynamics, Butterworth-Heinemann, Oxford 2008.

[10] E.D. Nascimento, O. Helene, V. Vanin, M.D. Cruz, and M. Moralles, Nucl. Instr. Meth. Phys. Res. A 609, 244 (2009).
[11] N.W. Ashcroft, N.D. Mermin, Solid State Physics, Brooks/Cole Thomson Learning, South Melbourne 2012.

[12] W. Lotz, J. Opt. Soc. Am. 60, 206 (1970). 